\title{
UCLA
}

\section{Recent Works}

\section{Title}

Metabolic repair through emergence of new pathways in Escherichia coli

Permalink

https://escholarship.org/uc/item/2t58m71t

\section{Journal}

Nature Chemical Biology, 14

\section{Authors}

Pontrelli, Sammy

Fricke, Riley C. B.

Teoh, Shao Thing

et al.

\section{Publication Date}

2018-11-01

Peer reviewed 


\title{
Metabolic repair through emergence of new pathways in Escherichia coli
}

\author{
Sammy Pontrelli $\odot^{1}$, Riley C. B. Fricke ${ }^{1}$ ', Shao Thing Teoh $\oplus^{2}$, Walter A. Laviña ${ }^{2,3}$, Sastia Prama Putri ${ }^{2}$, \\ Sorel Fitz-Gibbon ${ }^{(4}{ }^{4}$, Matthew Chung ${ }^{\circledR}{ }^{1}$, Matteo Pellegrini ${ }^{4,5}$, Eiichiro Fukusaki ${ }^{2}$ and James C. Liao ${ }^{6 *}$
}

\begin{abstract}
Escherichia coli can derive all essential metabolites and cofactors through a highly evolved metabolic system. Damage of pathways may affect cell growth and physiology, but the strategies by which damaged metabolic pathways can be circumvented remain intriguing. Here, we use a $\Delta$ panD (encoding for aspartate 1-decarboxylase) strain of $E$. coli that is unable to produce the $\beta$-alanine required for CoA biosynthesis to demonstrate that metabolic systems can overcome pathway damage by extensively rerouting metabolic pathways and modifying existing enzymes for unnatural functions. Using directed cell evolution, rewiring and repurposing of uracil metabolism allowed formation of an alternative $\beta$-alanine biosynthetic pathway. After this pathway was deleted, a second was evolved that used a gain-of-function mutation on ornithine decarboxylase (SpeC) to alter reaction and substrate specificity toward an oxidative decarboxylation-deamination reaction. After deletion of both pathways, yet another independent pathway emerged using polyamine biosynthesis, demonstrating the vast capacity of metabolic repair.
\end{abstract}

U nlike DNA, metabolic systems do not possess a repair mechanism. However, it has been proven that metabolism has the ability to overcome deleterious consequences caused by pathway damage when reactions of a metabolic pathway are blocked ${ }^{1-4}$. One means by which blocked enzymes can be complemented is with the use of isozymes, which may serve as spare parts ${ }^{5}$. Alternatively, promiscuous enzymes may be present to complement a lost function $^{2,6,7}$. Other enzymes are highly specialized and catalyze unique reactions, and when damaged they may compromise growth and alter physiology. The wide range of possible reactions catalyzed by cryptic and promiscuous enzymes have the potential to form pathways that can restore metabolic function when these specialized reactions are blocked $^{8}$. Although cells have proven the ability to bypass blocked metabolic function, the diverse mechanisms and extent to which the cell can bypass metabolic blocks have not been thoroughly explored. Here, we use a $\triangle$ panD (encoding for aspartate 1-decarboxylase) strain of E. coli, which is incapable of producing the $\beta$-alanine required for synthesis of $\mathrm{CoA}$, as an example to demonstrate the repair capabilities of cell metabolism.

Aspartate 1-decarboxylase (PanD) is the only enzyme capable of $\beta$-alanine synthesis in $E$. coli. In bacteria, fungi, and plants, $\beta$-alanine is a precursor to pantothenate, which in turn is a required metabolite for the synthesis of coenzyme A (CoA) in all organisms ${ }^{9}$. In animals, $\beta$-alanine is synthesized as a precursor to carnosine, which is found at high concentrations in skeletal muscle tissue and the central nervous system and is used for various physiological purposes ${ }^{10}$. Without CoA, the cell is incapable of carrying out essential cellular processes including the TCA cycle, fatty acid biosynthesis, and acetyl-CoA synthesis, which is used as a building block for many essential compounds $s^{9}$. Therefore, unless $\beta$-alanine or pantothenate are supplemented, a $\Delta$ panD strain cannot grow on minimal media alone. Unlike most decarboxylases that use pyridoxal-5' -phosphate (PLP) as a cofactor, PanD uses a covalently bound pyruvoyl group ${ }^{11}$.
PanD is first translated as an inactive protoenzyme that is cleaved into $\alpha$ - and $\beta$-subunits, which is triggered by the activator PanZ ${ }^{12}$. This likely serves as an additional regulatory element to control intracellular levels of pantothenate.

Several other pathways are believed to exist in other organisms to supply $\beta$-alanine: degradation of propionate into malonic semialdehyde (MSA) and subsequent transamination ${ }^{13}$, a reductive uracil degradation pathway using dihydrouracil as an intermediate ${ }^{14}$, and an oxidative degradation of spermine into $\beta$-alanine using 3 -aminopropanal as an upstream precursor ${ }^{15}$. However, within E. coli, these pathways are not known to exist. Here, we show that to overcome damage to the $\beta$-alanine pathway, E. coli metabolism can be repaired through the emergence of at least three novel metabolic pathways to produce $\beta$-alanine. Our results demonstrate the intrinsic pliability of biological systems.

\section{Results}

Evolution to overcome $\beta$-alanine auxotrophy. We first attempted to generate a $\Delta$ panD deletion strain to overcome $\beta$-alanine auxotrophy by repeatedly diluting cultures that contain limited amounts of $\beta$-alanine. This approach did not yield successful results after 20 serial dilutions. We then sought to enhance the rate of mutagenesis to obtain strains that overcome auxotrophy in a shorter timeframe. This was accomplished by overexpressing a mutator gene, mutD5 (ref. ${ }^{16}$ ), which compromises DNA proofreading in a dominant negative manner. Within 20 serial dilutions, 12 of 15 independent cultures overcame $\beta$-alanine auxotrophy with an average number of $225 \pm 134$ mutations per genome (Supplementary Dataset 1). Over the course of evolution, the concentration of $\beta$-alanine required to sustain growth of the cultures dropped more than two orders of magnitude (Supplementary Fig. 1). An isolated clonal strain, PS1, was further studied to investigate how the strain overcame $\beta$-alanine auxotrophy.

'Department of Chemical and Biomolecular Engineering, University of California, Los Angeles, Los Angeles, CA, USA. 'Department of Biotechnology, Graduate School of Engineering, Osaka University, Suita, Osaka, Japan. ${ }^{3}$ Microbiology Division, Institute of Biological Sciences, College of Arts and Sciences, University of the Philippines Los Baños, Los Baños, Laguna, Philippines. ${ }^{4}$ Institute of Genomics and Proteomics, University of California, Los Angeles, Los Angeles, CA, USA. '5 Department of Molecular, Cell, and Developmental Biology, University of California, Los Angeles, Los Angeles, CA, USA. ${ }^{6}$ Institute of Biological Chemistry, Academia Sinica, Taipei, Taiwan. *e-mail: Liaoj@gate.sinica.edu.tw 


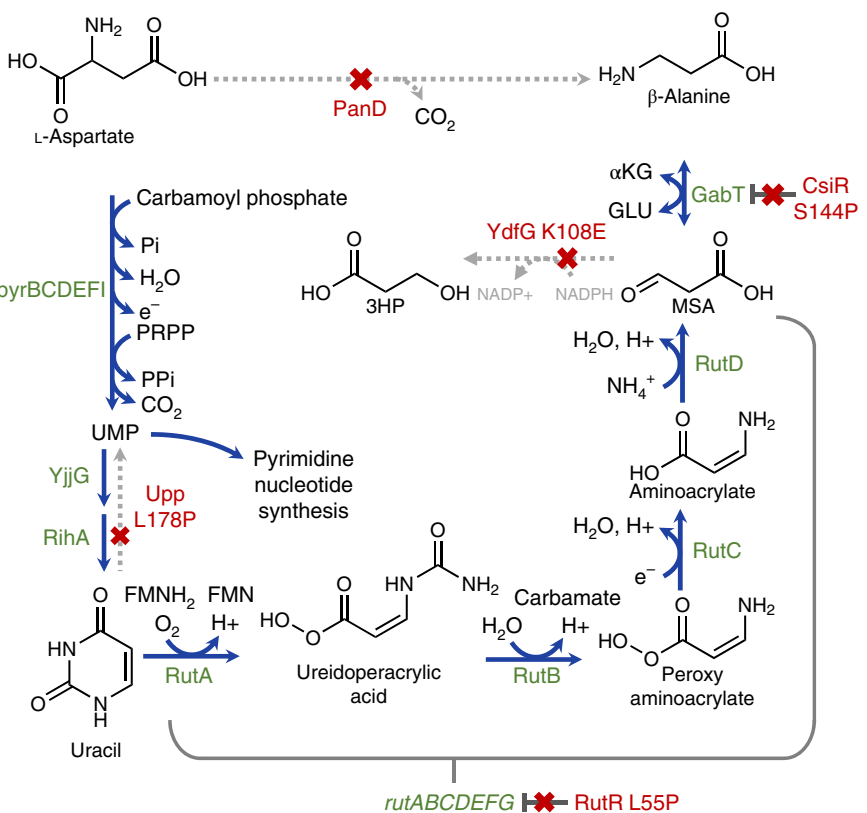

Fig. 1 | $\beta$-alanine biosynthesis through uracil degradation. A pathway emerged to overcome $\beta$-alanine auxotrophy that repurposed the uracil degradation pathway (encoded by rutABCDEFG) and GabT. Mutations that contribute to this phenotype are noted in red. MSA, malonic semialdehyde. UMP, uridine monophosphate.

Pathway emergence using uracil degradation. Interestingly, although many amino acid decarboxylases exist in E. coli, none emerged with function sufficient to directly complement PanD. Rather, the strain rerouted metabolism though pyrimidine synthesis and uracil degradation pathways (Fig. 1). This was accomplished solely through modulating enzyme expression levels rather than changing enzyme functions. Four essential mutations were identified that enabled this pathway rerouting. The first two mutations identified are on repressors RutR (L55P) and CsiR (S144P) (Fig. 1). These mutations enabled derepression of the pyrimidine utilization (Rut) pathway and 4-aminobutyrate transaminase (GabT), respectively (Fig. 2a). Together, these form the core enzymatic constituents of the pathway. The Rut pathway degrades uracil into 3-hydroxypropionic acid (3HP) as a terminal product ${ }^{17}$. However, GabT is a promiscuous transaminase that is known to convert MSA-the penultimate metabolite of the Rut pathway-into $\beta$-alanine ${ }^{18}$, and therefore is able to reroute the terminal product of the Rut pathway. Deletion of either RutA or GabT from PS1 abolishes the evolved phenotype, confirming their essential contributions (Fig. 2b).

The third essential mutation was acquired in the final enzyme of the Rut pathway ${ }^{17}$, 3-hydroxy acid dehydrogenase, YdfG (K108E), which diminishes, but does not abolish, the enzyme function (Fig. 2c). Although this mutation may serve to redirect the terminal product of the Rut pathway to $\beta$-alanine, deletion of YdfG from PS1 abolishes the evolved phenotype (Fig. 2b). Because MSA is a toxic intermediate ${ }^{17}$, a minor amount of YdfG activity may be essential to prevent toxic buildup. GabT catalyzes a thermodynamically reversible transamination between MSA and $\beta$-alanine, and we consequently observed toxicity caused by excess $\beta$-alanine supplementation (Fig. 2d). We demonstrated the ability of YdfG to relieve this toxicity as overexpression within PS1 restores growth when in the presence of excess $\beta$-alanine (Fig. 2d).

A fourth mutation was identified on Upp (L178P), uracil phosphoribosyltransferase, which completely abolishes the enzyme activity (Fig. 2e). Upp catalyzes the synthesis of uridine $5^{\prime}$-monophosphate (UMP), the precursor for all pyrimidine nucleotides,
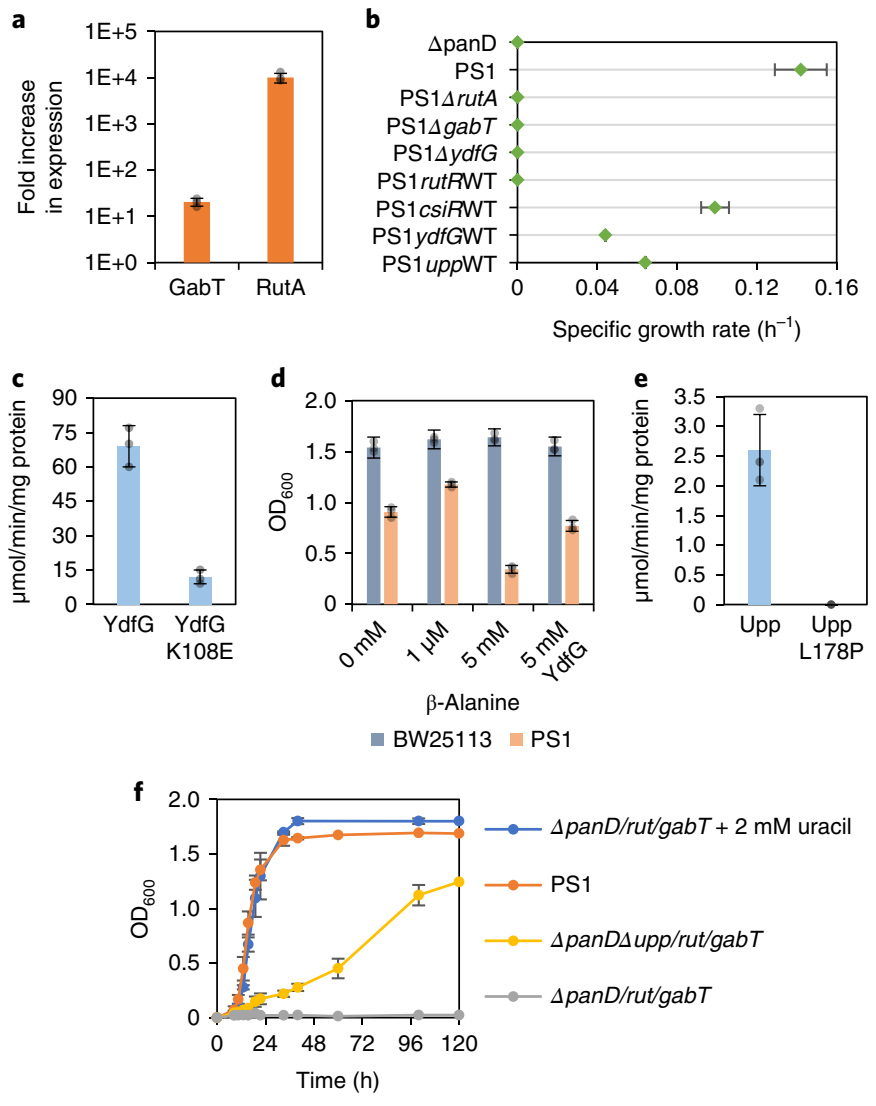

Fig. 2 | Characterization of mutations that contribute to the degradation of uracil into $\boldsymbol{\beta}$-alanine. a, RT-qPCR measurements of GabT and RutA in PS1 compared to wild-type parent strain BW25113 from $n=3$ biologically independent samples. b. Growth phenotypes of PS1 with deletion of pathway enzymes or reversion of point mutations to wild-type sequences. Point-mutation reversions for rutR, $c s i R, y d f G$ and upp are denoted as PS1rutRWT, PS1csiRWT, PS1ydfGWT, and PS1uppWT, respectively. Measurements were taken from $n=3$ biologically independent samples. c, In vitro specific activity of wild-type YdfG compared to YdfG K108E shows reduced activity from $n=3$ independent repeats. d, Growth of PS1 in minimal media with varying amounts of $\beta$-alanine supplementation shows that excess $\beta$-alanine is toxic to PS1. Overexpression of YdfG relieves this toxicity. Growth is measured from $n=3$ biologically independent samples. e, In vitro specific activity of wild-type Upp compared to Upp L178P shows complete loss of activity from $n=3$ independent repeats. $\mathbf{f}$, The evolved $\beta$-alanine pathway is reconstructed in an unevolved $\Delta$ panD strain. Overexpression of the rut operon and gabT in a $\Delta$ panD strain, denoted $\Delta$ panD/rut/gabT, can only rescue growth with uracil supplementation. Further deletion of upp ( $\Delta$ panD $\Delta u p p / r u t / g a b T$ ) eliminates the need for uracil supplementation. Growth was measured from $n=3$ biologically independent samples. All error bars represent s.d. of the mean. GabT is expressed from pALQ68, and the Rut pathway is expressed from pALQ78.

from uracil and 5-phospho- $\alpha$-D-ribose 1-diphosphate (PRPP). This reaction recycles the uracil formed as a degradation product from nucleic acids ${ }^{19}$. Intracellular concentrations of metabolites in PS1 and a $\triangle$ panD parent strain were compared and showed decreased concentrations of pyrimidine nucleotides within PS1 (Supplementary Fig. 2), suggesting that this mutation enables sufficient outflux of uracil into the Rut pathway.

We attempted to reconstruct the pathway in an unevolved $\Delta$ panD strain with only overexpression of the Rut operon and GabT. In this strain, additional uracil supplementation was required (Fig. 2f). However, when Upp was further deleted, growth was observed 


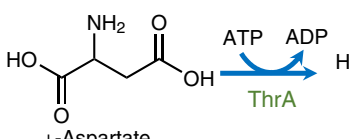

L-Aspartate

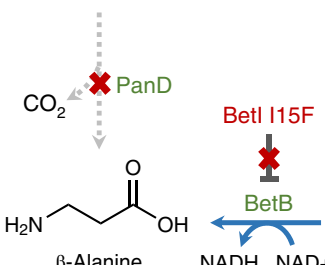

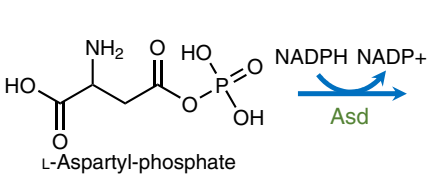
L-Aspartyl-phosphate

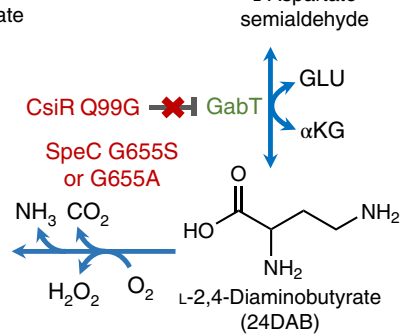

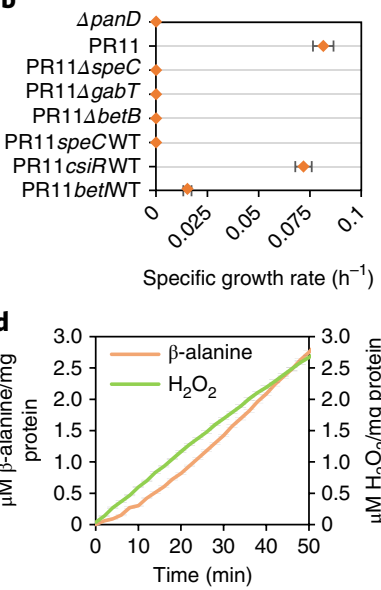

Time (min) c

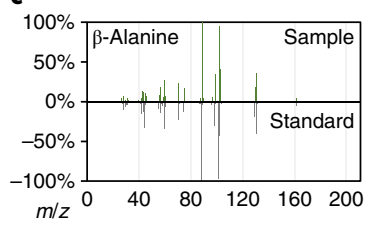

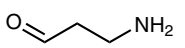

-Aminopropana (3AP)

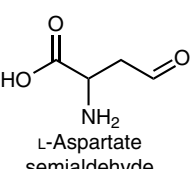

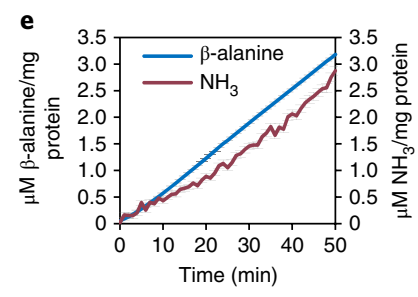

Fig. 3 | Emergence of $\boldsymbol{\beta}$-alanine synthesis pathway using evolved ornithine decarboxylase. $\mathbf{a}$, Illustration of the pathway that formed in PR11 to reroute damaged $\beta$-alanine biosynthesis. This pathway utilizes a gain of function mutation on ornithine decarboxylase (SpeC) that allows a decarboxylationdependent deamination reaction. Relevant mutations are noted in red. $\mathbf{b}$, Growth in minimal media of evolved strain PR11 with deletion of pathway enzymes or point-mutation reversions to wild-type sequences. PR11 with wild-type point-mutation reversions on speC, csiR, and betl are referred to as PR11speCWT, PR11csiRWT, and PR11bet/WT, respectively. Growth was measured from $n=3$ biologically independent samples. $\mathbf{c}$, Mass spectrum of $\beta$-alanine produced from 24DAB (2,4-diaminobutyrate) in vitro using a coupled assay with purified SpeC and BetB. This experiment was repeated three times with similar results. d,e, $\beta$-alanine formation with, $\mathrm{H}_{2} \mathrm{O}_{2}(\mathbf{d})$ and $\mathrm{NH}_{3}(\mathbf{e})$ measured from a coupled $\mathrm{SpeC} \mathrm{G} 655 \mathrm{~S}$ and BetB assay show equimolar formation of all products. Measurements were taken from $n=3$ independent repeats. All error bars represent s.d. of the mean.

without additional supplementation (Fig. 2f). When each of the four identified mutations were individually reverted in PS1, the evolved phenotype was either weakened or completely abolished (Fig. 2b).

To determine whether this same pathway formed in the remaining 11 evolved $\triangle$ panD strains, we deleted RutABC from each one. Deleting RutABC abolished the evolved phenotype of all but three strains, suggesting the existence of additional mechanisms of metabolic repair. Several genes exhibited a high prevalence of mutations on the genomes of each strain (Supplementary Table 1). Notably, YdfG was mutated in each strain, whereas RutR was mutated in five of nine evolved strains that use the uracil degradation pathway.

Pathway emergence with evolved ornithine decarboxylase. We next obtained strains that specifically overcame auxotrophy independent of uracil and the Rut degradation pathway. A double deletion $\triangle$ panD $\triangle$ rut $A B C$ strain was subjected to serial dilutions with limiting $\beta$-alanine and expression of mutator mutD5. After 85 dilutions, 3 of 12 independent cultures again overcame $\beta$-alanine auxotrophy. Genomes of all strains were sequenced to reveal an average of $149 \pm 36.1$ mutations per genome (Supplementary Dataset 1 ).

All double $\triangle p a n D \Delta r u t A B C$ strains with evolved phenotypes, in addition to the three remaining $\triangle$ panD evolved strains, acquired a mutation on the same residue of ornithine decarboxylase, SpeC (G655S or G655A) (Fig. 3a and Supplementary Table 2). Deletion of SpeC from any of these strains resulted in complete abolishment of the restored phenotype (Fig. 3b), demonstrating its essentiality. Reversion of the SpeC point mutation was tested in one strain (PR11) and abolished the growth phenotype (Fig. 3b).

While SpeC normally functions as an ornithine decarboxylase, L-2,4-diaminobutyrate (24DAB) is a structural homolog of L-ornithine. The analogous decarboxylation product of $24 \mathrm{DAB}$ is 1,3-propanediamine (13PDA), which may serve as an upstream precursor of $\beta$-alanine (Supplementary Fig. 3). We therefore hypothesized that SpeC acquired a mutation that allowed it to expand its substrate specificity to function using 24DAB. However, in vitro assays with purified enzyme revealed that $24 \mathrm{DAB}$ decarboxylase activity was detected from wild-type SpeC (Supplementary Fig. 3), but both mutated SpeC variants completely lost this activity.
Surprisingly, in the absence of 13PDA formation, 24DAB consumption was still observed from mutated SpeC variants in vitro. Similar PLP-dependent amino acid decarboxylases have reported side reactions that result in decarboxylation-dependent deamination of the $\alpha$-amino group to yield the respective aldehyde product $^{20,21}$. In this case, product formation from $24 \mathrm{DAB}$ yields 3 -aminopropanal (3AP), which can further be oxidized to $\beta$-alanine, possibly by betaine-aldehyde dehydrogenase (BetB) $)^{22}$ (Fig. 3a). A coupled in vitro assay of purified BetB and SpeC yielded $\beta$-alanine from 24DAB (Fig. 3c) with all SpeC variants. Previous reports of a decarboxylation-dependent deamination reaction involved a net reaction that required the consumption of dissolved oxygen and formation of ammonia and hydrogen peroxide ${ }^{20}$. We confirmed the presence of this reaction, as $\beta$-alanine formation was detected in a 1:1 molar ratio with production of both ammonia and hydrogen peroxide (Fig. 3d,e).

The native enzyme betaine-aldehyde dehydrogenase, BetB ${ }^{22}$, has been reported to have secondary activity for converting $3 \mathrm{AP}$ to $\beta$-alanine. Deletion of BetB abolishes the evolved phenotype, confirming its contribution (Fig. 3b). BetI, the corresponding repressor of BetB, is mutated in PR11 (BetI I15F), and a measured increase in expression of BetB demonstrates the deleterious consequence of this mutation (Fig. 4a). Reversion of the BetI mutation also diminishes the growth rate of PR11 (Fig. 3b). Upstream of these reactions in the pathway, we suspected that L-aspartate semialdehyde, a central metabolite essential for L-lysine, L-threonine, and L-methionine biosynthesis, may be a direct precursor of an aminotransferase reaction that can produce $24 \mathrm{DAB}$ directly (Fig. 3a). A mutation was found on CsiR (Q99G), the repressor of GabT, in PR11 to suggest its involvement. To verify that CsiR is deleteriously mutated, we measured GabT expression in PR11, revealing that it is in fact increased (Fig. 4a). GabT, which functions as a $\beta$-alanine aminotransferase in PS1, was determined to catalyze the $24 \mathrm{DAB}$ aminotransferase reaction needed in PR11 (Supplementary Fig. 4). To further confirm the pathway, we proceeded to reconstruct the complete pathway in an unevolved $\triangle$ panD strain. Overexpression of GabT, BetB, and SpeC G655S was sufficient to rescue growth in minimal media without $\beta$-alanine supplementation (Fig. $4 \mathrm{~b}$ ). Here, the identified mutations 

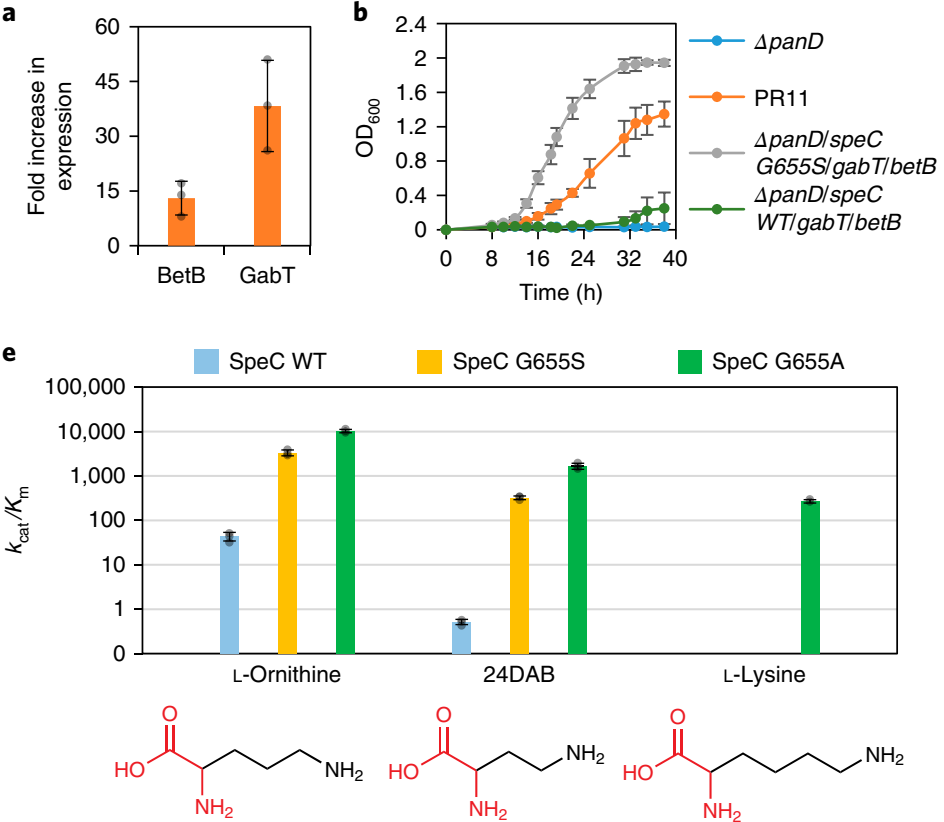

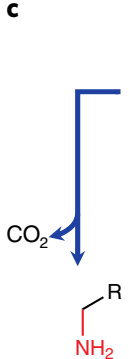

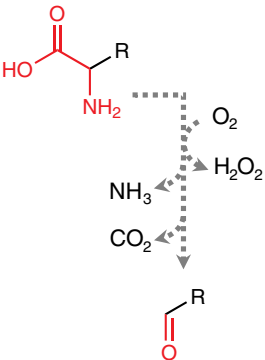

d

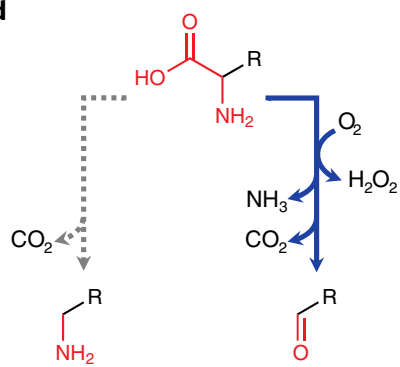

Fig. 4 I $\beta$-alanine pathway using SpeC and pathway reconstruction. a, Relative gene expression of BetB and GabT in PR11 compared to wild-type parent BW25113 measured using RT-qPCR. Measurements were taken from $n=3$ biologically independent samples. $\mathbf{b}$, Reconstruction of this pathway to rescue growth of a $\Delta$ panD strain in minimal media is accomplished with overexpression of GabT, BetB, and SpeC G655S ( $\Delta$ panD/speC G655S/gabT/betB) but not with SpeC WT ( $\Delta$ panD/speC WT/gabT/betB). Measurements were taken from $n=3$ independent repeats. $\mathbf{c}, \mathbf{d}$, Two reaction types are observed from ornithine decarboxylase (SpeC) variants on three amino acid substrates. One is a decarboxylation reaction, while the other is a decarboxylation dependent deamination reaction. Wildtype SpeC can perform the decarboxylation reaction on any of the three substrates, but only has minimal activity for the decarboxylation-dependent deamination reaction (c). SpeC G655S and G655A have high activity for decarboxylation-dependent deamination, but lost the ability to perform the decarboxylation on both 2,4-diaminobutyrate (24DAB) and L-lysine (d). The relevant functional groups are highlighted in red. e, Enzyme kinetics of the decarboxylation-dependent deamination reaction using purified SpeC variants in vitro. Measurements were taken from $n=3$ independent repeats. GabT and BetB are expressed from pALQ146, SpeC WT from pALQ142, and SpeC G655S from pALQ143. All error bars represent s.d. of the mean.

that contributed to metabolic repair increased expression of pathway constituents (CsiR and BetI) and altered activity (SpeC).

Activity of the SpeC variants using $24 \mathrm{DAB}$, L-ornithine, and L-lysine as substrates was tested in vitro. Whereas wild-type SpeC carries the decarboxylation-dependent deamination activity using L-ornithine as a substrate (Fig. 4c-e), this activity was almost undetectable with 24DAB. The mutations in SpeC (G655S or G655A) increased the $k_{\text {cat }}$ and decreased the $K_{\mathrm{m}}$ of this reaction for L-ornithine and 24DAB (Fig. 4c-e; Supplementary Table 3). G655A and G655S mutants presented 3,300- and 640 -fold $k_{\text {cat }} / K_{\mathrm{m}}$ increases, respectively, compared to $24 \mathrm{DAB}$ (Fig. 4e; Supplementary Table 3). This gain of function may be the key to the metabolic repair.

SpeC G655A is the onlyvariant capable of using all three substrates for a decarboxylation-dependent deamination reaction (Fig. 4e; Supplementary Fig. 5; Supplementary Table 3). On the other hand, wild-type SpeC is the only variant capable of a decarboxylation reaction on all three substrates to produce the corresponding polyamine product (Supplementary Fig. 6), whereas G655A and G655S variants completely lost the ability to perform this reaction on $24 \mathrm{DAB}$ and L-lysine. It appears that overcoming damage to the metabolic pathway takes advantage of the broader reactivity and substrate range made available from existing enzymes that may further evolve to have a highly specified function.

Additional pathway emergence. We sought to further test the ability of the cell to overcome metabolic pathway damage by constructing a $\Delta$ panD $\Delta$ rutABC $\Delta$ speC strain. With additional overexpression of mutD5, this strain was evolved for 45 dilutions to yield 1 of 12 strains that complemented $\beta$-alanine auxotrophy (Mel6, 372 mutations;
Supplementary Fig. 7; Supplementary Dataset 1). We were able to identify three essential enzymes: arginine decarboxylase (SpeA), $S$-adenosylmethionine decarboxylase (SpeD), and spermidine synthase (SpeE). Deletion of these genes from Mel6 abolished the evolved phenotype; further, an increase in expression was measured (Supplementary Fig. 8). These enzymes normally function to synthesize spermidine in E. coli, suggesting that an additional pathway may have formed that degrades polyamines into $\beta$-alanine using unknown enzymes. Observing the formation of an additional pathway by which $\beta$-alanine auxotrophy can be overcome illustrates the remarkable capability of the metabolic system to overcome damage using only endogenous genetic components.

Previous studies have tested the ability of $E$. coli to overcome a variety of metabolic auxotrophies ${ }^{1,3,4}$, and our results serve to validate these findings with specific mechanisms. To further determine the extent of the rerouting capability, we selected an additional 34 metabolic auxotrophs unable to grow in glucose minimal media (Supplementary Table 4), and subjected them to a minimum of 20 serial dilutions with limited nutritional supplementation or until the ability to grow without supplementation was acquired. These 34 mutants were chosen to represent deficiencies in a broad range of cofactor and amino acid biosynthetic pathways. Seven strains acquired the ability to grow without nutritional supplementation (Supplementary Fig. 9). These strains all recovered readily, requiring a small number of serial dilutions ( mean $=6.4$, Supplementary Fig. 9) and number of mutations (mean $=2.8$, Supplementary Dataset 1 ). We subjected the remaining 27 strains to additional rounds of evolution with overexpression of mutD5 to test whether increased mutagenesis can increase the number of strains that evolve. Of these 
strains, four (Supplementary Fig. 9) gained the ability to grow without nutritional supplementation with an average of $446.8 \pm 287.5$ mutations per genome (Supplementary Dataset 1). As expected, these results demonstrate that a more diverse set of metabolic deficiencies can be overcome with overexpression of $m u t D 5$.

\section{Discussion}

According to evolutionary theory, organisms survive external or internal challenges by acquiring mutations that give rise to new phenotypes. In metabolism, a new phenotype may be attributed to a new enzymatic function or a new pathway. In the simplest cases, adaptations enabling survival may involve upregulation of promiscuous enzymes or gene duplications followed by evolution of altered functions. Here we report a more complicated situation, in which new metabolic pathways are evolved to enable survival. The evolution of a new pathway, and rerouting of the metabolic flux through a new pathway, may require multiple mutations that upregulate the desired pathway, downregulate divergent pathways, and modulate enzymes that may affect toxic intermediates. In more complicated situations, altering enzyme functions to enable unnatural reactions may be required to form a novel pathway together with promiscuous enzymes. These cases are more difficult to observe in the laboratory.

Here we report the laboratory evolution of two independent metabolic pathways that enable the survival of E. coli in minimal medium after the deletion of panD. The first pathway involves extensive rerouting of metabolic flux through the Rut pathway. The second involves the mutation of $\mathrm{SpeC}$ to acquire a non-native function, together with upregulation of promiscuous enzymes to form a novel pathway. We demonstrated that there is at least one other pathway independent of Rut and SpeC that can complement the deletion of panD. These examples demonstrate that the cell is innately equipped with a vast set of endogenous genetic components that offer the potential to bypass damaged metabolic pathways. The fact that alternative pathways to provide $\beta$-alanine were repeatedly evolved demonstrates the vast capacity of metabolism to circumvent blockage. As such, metabolic repair poses a challenge for the development of robust engineered phenotypes and metabolic targeted drugs. However, it also serves as an opportunity for the emergence of new biochemical pathways that can further be exploited in engineering microbes for bioproduction.

\section{Online content}

Any methods, additional references, Nature Research reporting summaries, source data, statements of data availability and associated accession codes are available at https://doi.org/10.1038/ s41589-018-0149-6.

Received: 29 May 2018; Accepted: 13 September 2018; Published online: 16 October 2018

\section{References}

1. Patrick, W. M., Quandt, E. M., Swartzlander, D. B. \& Matsumura, I. Multicopy suppression underpins metabolic evolvability. Mol. Biol. Evol. 24, 2716-2722 (2006)

2. Notebaart, R. A., Kintses, B., Feist, A. M. \& Papp, B. Underground metabolism: network-level perspective and biotechnological potential. Curr. Opin. Biotechnol. 49, 108-114 (2018).

3. McLoughlin, S. Y. \& Copley, S. D. A compromise required by gene sharing enables survival: implications for evolution of new enzyme activities. Proc. Natl Acad. Sci. USA 105, 13497-13502 (2008).

4. Blank, D., Wolf, L., Ackermann, M. \& Silander, O. K. The predictability of molecular evolution during functional innovation. Proc. Natl Acad. Sci. USA 111, 3044-3049 (2014).

5. Reed, J. L., Vo, T. D., Schilling, C. H. \& Palsson, B. O. An expanded genome-scale model of Escherichia coli K-12 (i JR904 GSM/GPR). genome Biol. 4, R54 (2003).
6. Guzmán, G. I. et al. Model-driven discovery of underground metabolic functions in Escherichia coli. Proc. Natl Acad. Sci. USA 112, 929-934 (2015).

7. Khersonsky, O. \& Tawfik, D. S. Enzyme promiscuity: a mechanistic and evolutionary perspective. Annu. Rev. Biochem. 79, 471-505 (2010).

8. Kim, J., Kershner, J. P., Novikov, Y., Shoemaker, R. K. \& Copley, S. D. Three serendipitous pathways in E. coli can bypass a block in pyridoxal-5' -phosphate synthesis. Mol. Syst. Biol. 6, 436 (2010).

9. Webb, M. E., Smith, A. G. \& Abell, C. Biosynthesis of pantothenate. Nat. Prod. Rep. 21, 695-721 (2004).

10. Boldyrev, A. A. Carnosine: new concept for the function of an old molecule. Biochemistry (Mosc). 77, 313-326 (2012).

11. Joanne, W. M. \& Brown, G. M. Purification and properties of l-aspartate- $\alpha$ -decarboxylase, an enzyme that catalyzes the formation of $\beta$-alanine in Escherichia coli. J. Biol. Chem. 254, 8074-8082 (1979).

12. Nozaki, S., Webb, M. E. \& Niki, H. An activator for pyruvoyl-dependent $\mathrm{L}$-aspartate $\alpha$-decarboxylase is conserved in a small group of the $\gamma$-proteobacteria including Escherichia coli. MicrobiologyOpen 1, 298-310 (2012).

13. Rathinasabapathi, B. Propionate, a source of $\beta$-alanine, is an inhibitor of $\beta$-alanine methylation in Limonium latifolium, Plumbaginaceae. J. Plant Physiol. 159, 671-674 (2002).

14. Fritzson, P. The catabolism of C14-labeled uracil, dihydrouracil, and $\beta$ -ureidopropionic acid in rat liver slices. J. Biol. Chem. 226, 223-228 (1957).

15. White, W. H., Gunyuzlu, P. L. \& Toyn, J. H. Saccharomyces cerevisiae is capable of de novo pantothenic acid biosynthesis involving a novel pathway of beta-alanine production from spermine. J. Biol. Chem. 276, 10794-10800 (2001).

16. Maruyama, M., Horiuchi, T., Maki, H. \& Sekiguchi, M. A dominant (mut D5) and a recessive (dnaQ49) mutator of Escherichia coli. J. Mol. Biol. 167, 757-771 (1983).

17. Kim, K. S. et al. The Rut pathway for pyrimidine degradation: novel chemistry and toxicity problems. J. Bacteriol. 192, 4089-4102 (2010).

18. Borodina, I. et al. Establishing a synthetic pathway for high-level production of 3-hydroxypropionic acid in Saccharomyces cerevisiae via $\beta$-alanine. Metab. Eng. 27, 57-64 (2015).

19. Andersen, P. S., Smith, J. M. \& Mygind, B. Characterization of the upp gene encoding uracil phosphoribosyltransferase of Escherichia coli K12. Eur. J. Biochem. 204, 51-56 (1992).

20. Bertoldi, M., Carbone, V. \& Borri Voltattorni, C. Ornithine and glutamate decarboxylases catalyse an oxidative deamination of their $\alpha$-methyl substrates. Biochem. J. 342, 509-512 (1999).

21. Kaminaga, Y. et al. Plant phenylacetaldehyde synthase is a bifunctional homotetrameric enzyme that catalyzes phenylalanine decarboxylation and oxidation. J. Biol. Chem. 281, 23357-23366 (2006).

22. Incharoensakdi, A. et al. Overproduction of spinach betaine aldehyde dehydrogenase in Escherichia coli. Structural and functional properties of wild-type, mutants and E. coli enzymes. Eur. J. Biochem. 267, 7015-7023 (2000).

\section{Acknowledgements}

This research was supported in part by a grant from National Science Foundation (MCB 1139318) for JP-US "Metabolomics for Low Carbon Society" (received by J.C.L.), and Japan Science and Technology's Strategic International Collaborative Research Program (received by E.F). S.F.-G. acknowledges support from a QCB Collaboratory Postdoctoral Fellowship, and the QCB Collaboratory community directed by M. Pellegrini.

\section{Author contributions}

J.C.L. and S.P. conceived the idea of this project and wrote the manuscript. S.P. designed all experiments and performed evolution, RT-qPCR, enzyme assays, gene deletions, genome sequencing library generation, mass spec verification and measured growth phenotypes. R.C.B.F. performed evolution, enzyme assays, point mutation reversions, and growth curve analysis. S.T.T., W.A.L., S.P.P. and E.F. performed metabolomic analysis and data analysis. M.C. performed evolution. S.F.-G. and M.P. performed genomic sequencing and analysis.

\section{Competing interests}

The authors declare no competing interests.

\section{Additional information}

Supplementary information is available for this paper at https://doi.org/10.1038/ s41589-018-0149-6.

Reprints and permissions information is available at www.nature.com/reprints.

Correspondence and requests for materials should be addressed to J.C.L.

Publisher's note: Springer Nature remains neutral with regard to jurisdictional claims in published maps and institutional affiliations.

(c) The Author(s), under exclusive licence to Springer Nature America, Inc. 2018 


\section{Methods}

Strains, reagents, and plasmids. All chemicals and reagents were purchased from Sigma-Aldrich unless otherwise noted. Escherichia coli single knockouts strains were obtained from the Keio collection ${ }^{23}$, and parent strain BW25113 was obtained from Thermo-scientific.

Primers were purchased through Integrated DNA Technologies (http://IDTdna. com). RBS sequences were optimized using RBS Calculator v2.0 (refs. ${ }^{24,25}$ ). All PCR reactions were first performed using KOD Hot-Start DNA polymerase (EMD Millipore). If these reactions failed, reactions were repeated with KOD Xtreme Hot-Start Polymerase (EMD Millipore). Assembly of fragments was performed using T4 DNA polymerase (NEB) using the following protocol: Gel purified PCR fragments with 20-bp overlaps were mixed in equimolar amounts. Approximately $300 \mathrm{ng}$ of the fragment mixture was combined with NEB buffer \#2 and $0.3 \mu$ $1 \mathrm{~T} 4$ polymerase to a final volume of $10 \mu \mathrm{l}$. The reaction was incubated at room temperature $\left(20-22^{\circ} \mathrm{C}\right)$ for $5 \mathrm{~min}$, transformed into $E$. coli XL1Blue (Agilent), and selected on LB agar plates containing appropriate antibiotics. Gene deletions on strains were carried out using $\lambda$ Red recombinase as previously described ${ }^{26}$. MutD5 gene was obtained by amplifying DnaQ from XL1Red strains (Agilent). His-tagged proteins are cloned and expressed within pCDFDuet backbones (Addgene). His tagged plasmids were constructed using primers described in Supplementary Table 3 and 4. All strains and plasmids are listed in Supplementary Table 5.

Growth conditions. Growth curves for PS1, PR11 and Mel6 derived strains were obtained within cultures of glucose M9 minimal media: M9 Minimal Salts (Fisher Scientific), $0.4 \%$ glucose, $1 \mathrm{mM} \mathrm{MgSO}_{4}, 0.1 \mathrm{mM} \mathrm{CaCl}_{2}, 1 \mathrm{mg} / \mathrm{L}$ thiamine. Antibiotics were used at the following concentrations: kanamycin sulfate $50 \mu \mathrm{g} / \mathrm{ml}$, disodium carbenicillin (Gold Biotechnology) $100 \mu \mathrm{g} / \mathrm{ml}$, chloramphenicol $20 \mu \mathrm{g} /$ $\mathrm{ml}$, and spectinomycin (Gold Biotechnology) $100 \mu \mathrm{g} / \mathrm{ml}$.

Optical density was measured using Agilent 8453 Spectrophotometer. During phenotypic analysis of PS1, PR11 and Mel6 (and derivatives), colonies were inoculated into $3 \mathrm{ml}$ glucose minimal media containing limiting concentrations of $\beta$-alanine $(1 \mu \mathrm{M})$ and grown for $24 \mathrm{~h}$ at $37^{\circ} \mathrm{C}$. Cells were used in a 1:1,000 inoculation into selection media containing appropriate supplements unless otherwise noted. Growth curves and specific growth rates were measured from three independent cultures grown in parallel.

Evolution of suppressor phenotypes. Strains were evolved to overcome various auxotrophies by gradually reducing the availability of nutritional supplementation. Mutant strains were inoculated into a preculture of LB containing an appropriate antibiotic, grown overnight at $37^{\circ} \mathrm{C}$, and further used to inoculate $1: 100$ into minimal medium with a respective carbon source and antibiotic. Strains were passed into fresh minimal media at a 1:1,000 dilution with limiting supplementation sufficient to maintain growth between $\mathrm{OD}_{600} 0.4$ and 0.6 . These cultures were diluted daily. If growth exceeded $\mathrm{OD}_{600} 0.6$, the amount of nutritiona supplementation was reduced two-fold for the next dilution. If cultures grew past $\mathrm{OD}_{600} 1.0$, they were passed into minimal media without further supplementation. If this culture grew, they were streaked onto minimal media agar plates without supplementation. Genotypes of growing colonies were verified using PCR and their phenotype was reconfirmed by inoculating directly from the plate into minimal media. Unless otherwise specified, four independent cultures of each strain were subjected to serial dilutions.

Within certain strains, mutation rates were accelerated with use of a mutator plasmid that expresses MutD5. MutD5 (ref. ${ }^{27}$ ) is a dominant negative mutant of DNA polymerase III subunit $\varepsilon$, DnaQ, which catalyzes the $3^{\prime}$ to $5^{\prime}$ proofreading during DNA replication ${ }^{28}$.

Strains were either evolved for a minimum of 20 dilutions or until the ability to grow in minimal media without supplementation was obtained. Occasionally, the addition of a mutator plasmid into a given strain results in the loss of growth within minimal media even with nutritional supplementation. If this occurred, the strain was no longer subjected to serial dilutions.

Curing plasmids. Plasmids were cured using acridine orange. Overnight cultures were made in LB without antibiotics. The following day, $1 \%$ inoculations were made into fresh LB cultures containing $2 \mu \mathrm{l}$ of acridine orange (Thermo Fisher) for every $1 \mathrm{ml}$ of LB. These were grown overnight at $42{ }^{\circ} \mathrm{C}$, streaked onto LB plates, and then screened for the correct lack of antibiotic resistance. The lack of plasmid was then confirmed through PCR verification.

Quantification of amino acids and polyamines using HPLC. Amino acids and polyamines were derivatized using OPA and FMOC reagents (Agilent) and analyzed using a Zorbax Eclipse AAA HPLC column $(5 \mu \mathrm{M}$ beads, $4.6 \mathrm{~mm} \times 150 \mathrm{~mm})$. All protocols were obtained from Agilent.

GC-MS analysis of MCF derivatives. All columns and instruments were purchased from Agilent Technologies. GC-MS data was obtained using a 6890/5973 GC-MS and DB-624UI (GC-MS) column. The oven temperature was initially set at $60^{\circ} \mathrm{C}$ for $2 \mathrm{~min}$. Then a ramping of $16^{\circ} \mathrm{C} / \mathrm{min}$ was applied to a gradient reaching $180^{\circ} \mathrm{C}$, followed by a $3 \mathrm{~min}$ hold. Next, a $40^{\circ} \mathrm{C} / \mathrm{min}$ ramping was applied to a gradient reaching $220^{\circ} \mathrm{C}$, followed by a $3 \mathrm{~min}$ hold. The $40^{\circ} \mathrm{C} / \mathrm{min}$ ramping was again applied to a temperature of $240{ }^{\circ} \mathrm{C}$, followed by a 6 min hold. The flow through the column was held constant at $1.8 \mathrm{ml} \mathrm{He} / \mathrm{min}$. The injection volume was $2 \mu \mathrm{l}$ and the split ration $20: 1$. The temperature of the inlet was $180^{\circ} \mathrm{C}$, and the detector was set to $250^{\circ} \mathrm{C}$.

Preparation of cell lysate for enzyme assays and protein purification. Respective plasmids were transformed into E. coli BL-21 DE3 (Invitrogen) and grown overnight. These cultures were used for a $1 \%$ inoculation into LB, grown to midlog phase, and were induced using $0.1 \mathrm{mM}$ isopropyl-h-D-thiogalactopyranoside (IPTG) overnight at $30^{\circ} \mathrm{C}$. Cells were harvested by centrifugation and lysed using Qiagen TissueLyser II. His-tagged proteins were purified using HisPur Ni-NTA Spin Purification Kit (Thermo Fisher).

YdfG assay. Plasmids pALQ200 and pALQ201 were used to express 5' his-tagged wild-type and mutant (K108E) enzymes, respectively. These were further purified using HisPur Ni-NTA Spin Purification Kit (Thermo Fisher). Standard reaction mixture was composed of $50 \mathrm{mM}$ Tris- $\mathrm{HCl}$ buffer ( $\mathrm{pH} 8.5$ ), $0.5 \mathrm{mM} \mathrm{NADP}^{+}$and $5 \mathrm{mM} 3$-hydroxypropionic acid. The reaction was started by adding $10 \mu \mathrm{l}$ of cell lysate to $200 \mu \mathrm{l}$ of reaction mixture and monitoring $\mathrm{OD}_{340}$ increase over time using Bio-TEK Powerwave XS plate reader.

Upp assay. Plasmids pALQ198 and pALQ199 were used to express and purify 5 his-tagged wild-type and mutant (L178P), respectively. This assay was adapted from previous study ${ }^{29}$. Standard reaction mixture was composed of $50 \mathrm{mM}$ Tris- $\mathrm{HCl}$ buffer (pH 8.5), 1 mM GTP (Sigma), 2 mM PRPP (Sigma), 2 mM uracil (Sigma), and $50 \mathrm{mM} \mathrm{MgCl}_{2}$. Cell lysate was normalized to $1.5 \mathrm{mg} / \mathrm{ml}$ using Bradford Reagent. Reaction mixture was started by adding $50 \mu \mathrm{l}$ of lysate to $1 \mathrm{ml}$ of reaction buffer. The reaction was stopped by the addition of $80 \mu$ l of reaction mixture to $20 \mu \mathrm{l}$ of ice cold $100 \% \mathrm{w} / \mathrm{v}$ trichloroacetic acid and vortexed. The mixture was kept at $-20^{\circ} \mathrm{C}$ for $6 \mathrm{~h}$ and centrifuged at maximum speed at $4{ }^{\circ} \mathrm{C}$; the supernatant was then collected. Uracil consumption was measured using highperformance liquid chromatography (HPLC) previously described ${ }^{30}$.

SpeC assay. Assay for SpeC is adapted from a previous study ${ }^{31}$. Plasmids pALQ142, pALQ143, and pALQ173 were used to express and purify $5^{\prime}$ his-tagged SpeC variants G655S, wild-type, and G655A, respectively. Protein was purified using HisPur Ni-NTA Spin Purification Kit (Thermo Fisher). Standard reaction mixture contained $100 \mathrm{mM}$ PBS (pH 8), 1 mM DTT, $1 \mathrm{mM} \mathrm{GTP,} 100 \mu \mathrm{M}$ PLP and $20 \mathrm{mM}$ L-ornithine, 2,4-diaminobutyrate or L-lysine. Reactions were initiated by addition of $30 \mu \mathrm{l}$ of enzyme to $1 \mathrm{ml}$ of enzyme mixture to reach a final concentration around $0.05 \mathrm{mg} / \mathrm{ml}$. Concentrations were later normalized using Bradford reagent. Reactions were allowed to proceed overnight and were stopped using Whatman Mini-UniPrep Nylon Syringeless Filters. Quantification of consumption of 2,4-diaminobutyrate was measured by HPLC using ZORBAX Eclipse AAA column. Samples were derivatized using OPA and FMOC reagents (Agilent) ${ }^{32}$. Product formation was later confirmed after MCF derivatization and analysis using GC-MS.

GabT assay for 2,4-diaminobutyrate transaminase. His-tagged GabT was overexpressed from pALQ144 and purified using HisPur Ni-NTA Spin Purification Kit (Thermo Fisher). The standard reaction mixture contained $100 \mathrm{mM}$ Tris- $\mathrm{HCl}$ buffer (pH 8), $100 \mu \mathrm{M}$ PLP, $20 \mathrm{mM}$ 2,4-diaminobutyrate, $20 \mathrm{mM} \alpha$-ketoglutarate and $1 \mathrm{mM}$ DTT. The reaction was allowed to proceed overnight at room temperature and was stopped using Whatman Mini-UniPrep Nylon Syringeless Filters. Formation of glutamate was measured using ZORBAX Eclipse AAA column on HPLC and was further derivatized using MCF derivatization and confirmed on GC-MS

Coupled SpeC and BetB assay. Decarboxylase and oxidative deaminase activity of SpeC was measured in a coupled reaction with BetB. The reaction mixture contained the following components: $25 \mu \mathrm{g} / \mathrm{ml}$ purified SpeC, $50 \mu \mathrm{g} / \mathrm{ml}$ purified BetB, $1 \mathrm{mM}$ GTP, $1 \mathrm{mM}$ NAD +, $100 \mu \mathrm{M}$ PLP, $20 \mathrm{mM}$ 24DAB or L-ornithine, and $1 \mathrm{mM}$ DTT in $100 \mathrm{mM} \mathrm{pH}$ 8.0 PBS. Increase in $\mathrm{OD}_{340}$ was measured over time at 37 ${ }^{\circ} \mathrm{C}$. When identifying product formation, the reaction was filtered using Whatman Mini-UniPrep Nylon Syringeless Filters, derivatized using MCF derivatization, then analyzed using GC-MS. Enzyme kinetics were measured using substrate concentrations of $0.5 \mathrm{mM}$ to $50 \mathrm{mM}$ for wild-type SpeC enzyme with all substrates, $1 \mu \mathrm{M}$ to $100 \mu \mathrm{M}$ for mutant SpeC enzymes with L-ornithine as a substrate, and $0.1 \mathrm{mM}$ to $1 \mathrm{mM}$ for mutant SpeC enzymes with 2,4-diaminobutyrate and L-lysine as substrates. The kinetic parameters $k_{\text {cat }}$ and $K_{\mathrm{m}}$ were determined by fitting data to Lineweaver-Burk plots. Initial velocities were determined to be the period in which a linear increase in $\mathrm{OD}_{340}$ was observed with respect to time, during which less than $15 \%$ of the substrate was consumed.

Hydrogen peroxide assay. Hydrogen peroxide formation was measured simultaneously with $\beta$-alanine formation in a reaction coupling purified SpeC, BetB, and peroxidase from horseradish (Sigma). The peroxidase reacts with $\mathrm{H}_{2} \mathrm{O}_{2}$, phenol, and 4-aminoantipyrine to produce a colorimetric readout at $\mathrm{OD}_{505}\left(\mathrm{ref}^{21}\right)$. 3-Aminopropanal was oxidized into $\beta$-alanine using BetB with a simultaneous 
conversion of $\mathrm{NAD}^{+}$into $\mathrm{NADH}$, producing a readout at $340 \mathrm{~nm}$. The reaction mixture contained $25 \mu \mathrm{g} / \mathrm{ml}$ SpeC G655S, $50 \mu \mathrm{g} / \mathrm{ml} \mathrm{BetB,} 1 \mathrm{mM}$ GTP, $1 \mathrm{mM}$ DTT, $100 \mu \mathrm{M}$ PLP, 1 mM NAD $+20 \mathrm{mM} 24 \mathrm{DAB}, 4 \mathrm{mM}$ phenol, $6 \mathrm{mM}$ 4-aminoantipyrine, and approximately $100 \mathrm{U} / \mathrm{ml}$ peroxidase, in $\mathrm{PBS} \mathrm{pH} 8.0100 \mathrm{mM}$. The reaction was allowed to proceed at $37^{\circ} \mathrm{C}$.

Ammonia assay. Ammonia formation was measured by coupling purified SpeC with glutamate dehydrogenase (Sigma). SpeC G655S uses GTP as an activator; however, we observed that GTP inhibits the activity of glutamate dehydrogenase. Therefore, we used CTP as an activator, as it has previously been demonstrated to also activate $\mathrm{SpeC}^{33}$. The reaction mixture contained $25 \mu \mathrm{g} / \mathrm{ml}$ SpeC G655S, $4 \mathrm{U} /$ $\mathrm{ml}$ glutamate dehydrogenase, $5 \mathrm{mM} 24 \mathrm{DAB}, 1 \mathrm{mM}$ CTP, $1 \mathrm{mM}$ DTT, $100 \mu \mathrm{M}$ PLP, $20 \mathrm{mM} \alpha \mathrm{KG}$, and $1 \mathrm{mM} \mathrm{NADH}$. The reaction was allowed to proceed at $37^{\circ} \mathrm{C}$, and $\mathrm{OD}_{340}$ was measured over time.

MCF derivatization. Samples used for GC-MS analysis were derivatized using methylchloroformate (MCF) derivatization adapted from a previously reported protocol $^{34} .100 \mu \mathrm{l}$ of sample was added to $100 \mu \mathrm{l}$ of $2 \mathrm{M} \mathrm{NaOH}$. $167 \mu \mathrm{l}$ of methanol was added, followed by $34 \mu \mathrm{l}$ of pyridine and $20 \mu \mathrm{l}$ of MCF, and the sample was vortexed for $30 \mathrm{~s}$. Immediately, another $20 \mu \mathrm{l}$ of MCF was added, which was followed by another $30 \mathrm{~s}$ of vortexing. $400 \mu \mathrm{l}$ of chloroform was added, followed by an additional $10 \mathrm{~s}$ of vortexing. Next, $400 \mu \mathrm{l}$ of $50 \mathrm{mM}$ sodium bicarbonate was added, which was followed by $10 \mathrm{~s}$ of vortexing. A glass Pasteur pipette was used to remove the top phase, and $100 \mathrm{mg}$ of anhydrous sodium sulfate was added to the chloroform solution to bind the remaining water. A glass Pasteur pipette was used to transfer the entire dried chloroform solution to a glass vial for injection into the GC-MS. Samples that were not immediately analyzed were stored at $-80^{\circ} \mathrm{C}$.

Genomic sequencing. Genomic DNA was extracted from relevant samples using Qiagen DNeasy Blood \& Tissue Kit. All samples were diluted to $0.2 \mathrm{ng} / \mu \mathrm{l}$ using a Qubit measurements. This was used as input sample for Nextera XT sample preparation (Illumina). Final libraries were eluted in Qiagen EB buffer with $0.1 \%$ Tween 20 . The individual sample libraries were normalized to equimolar concentrations and diluted to $10 \mathrm{nM}$ solution. Samples were sequenced using 100 bp s.e.m. reads on an Illumina HiSeq2000 sequencing system with single end reads to a minimum of $10 \times$ coverage, average $=42 \times$ coverage.

Sequencing data analysis. Adaptor sequences were removed from reads using Trim Galore! (http://www.bioinformatics.babraham.ac.uk/) with quality trimming turned off. Trimmed reads were mapped using BWA-MEM v.0.7.12-r1039

(ref. ${ }^{35}$ ) to the Escherichia coli str. K-12 substr. MG1655 genome (NCBI Accession NC_000913). Variant discovery and filtering was done with GATK v 3.7-0-gcfedb67 (ref. ${ }^{36}$ ) using HaplotypeCaller in GVCF mode with ploidy 1 and ploidy 3 as needed, followed by GenotypeGVCFs, and finally VariantFiltration setting a minimum QD of 2 . SnpEff ${ }^{37}$ was used to determine the context of the variants and predict the functional impact. Additional custom scripts were used to identify variants of interest.

Reverse transcriptase quantitative PCR. Genomic DNA was purified using Qiagen DNeasy Blood and Tissue Kit for initial determination of primer efficiency using Luna Universal qPCR Master Mix. The DNA was initially diluted to approximately $1 \mathrm{ng} / \mu \mathrm{l}$ and three additional subsequent serial dilutions were used to determine primer efficiency. Primers were designed using Primer3 (ref. ${ }^{38}$ ) software to have annealing temperatures at $60^{\circ} \mathrm{C}$ and product sized between 75 and $150 \mathrm{bp}$ within their respective target gene. Primers are listed below in Supplementary Table 6 and are verified to have efficiency between 90 and 105\%. Gene frr, ribosome-recycling factor, was used as a reference gene ${ }^{39}$.

RNA was purified from cultures of glucose minimal media at $\mathrm{OD}_{600} 0.4-0.6$ These cultures were inoculated at $1 \%$ with precultures originating from glucose minimal media with $1 \mu \mathrm{M} \beta$-alanine. Approximately $2 \times 10^{8}$ cells were added to two volumes of Qiagen RNAprotect Bacteria Reagent, vortexed and centrifuged. $200 \mu \mathrm{l}$ of $15 \mathrm{mg} / \mathrm{ml}$ of lysozyme with $30 \mu \mathrm{l}$ of Proteinase K (NEB) was added, and the mixture was shaken at room temperature for $45 \mathrm{~min}$. RNeasy RNA purification protocol was followed with additional DNase Digestion (Qiagen) step to remove genomic DNA. RT-qPCR was performed using Luna Universal One-Step RT-qPCR Kit and results were analyzed using Bio-Rad CFX Manager 2.0.

Genomic point mutations. Point mutations were introduced using $\lambda$-red recombinase system ${ }^{26}$. Linear fragments were designed similar to those used for gene deletions, which amplify a kanamycin cassette from pKD13 that have $50 \mathrm{bp}$ overlaps intended for site specific homologous recombination. However, to introduce a point mutation, one of the overlaps was extended $400-1,000 \mathrm{bp}$ and contained a mutation that can be introduce in the genome when recombined. Here, the overlap contained a wild-type sequence, so that when the cassette is recombined several hundred base pairs upstream or downstream of a mutated gene, a point mutation will be repaired.

Briefly, primers were designed that amplify a kanamycin cassette from pKD13 (Supplementary Table 7). A 400-1,000 bp overlap was also amplified from a wild-type BW25113 genome (Supplementary Table 8). These were attached using
SOE PCR. The target strain, harboring pKD46, was grown overnight at $30^{\circ} \mathrm{C}$ with ampicillin. The next morning, it was inoculated $1 \%$ into fresh LB containing ampicillin and $1 \mathrm{mM} \mathrm{L}$-arabinose and grown until $\mathrm{OD}_{600}$ of 0.6 . This was then washed three times in ice-cold $10 \%$ glycerol. $400 \mathrm{ng}$ of linear fragment was added, and the reaction was electroporated. The strain was rescued at $37^{\circ} \mathrm{C}$ for $1 \mathrm{~h}$ and then plated onto kanamycin plates. Successful colonies that formed were sequenced around the area of integration using Sanger sequencing to verify successful point mutation reversion.

Metabolomic analysis. Cultures grown until $\mathrm{OD}_{600}=\sim 1$ were harvested for metabolome analysis by fast filtration of $10 \mathrm{ml}$ culture broth through PTFE membrane filters (pore size $0.45 \mu \mathrm{m}$, diameter $47 \mathrm{~mm}$; Millipore, MA, USA). The filter-bound cells were transferred to $2 \mathrm{ml}$ tubes and flash-frozen in liquid nitrogen to quench metabolism before storage at $-80^{\circ} \mathrm{C}$. Metabolite extraction was performed by adding $1.8 \mathrm{ml}$ extraction solvent (methanol/water/chloroform in $5: 2: 2$ ratio, additionally spiked with $20 \mu \mathrm{g} / \mathrm{ml} \mathrm{ribitol} \mathrm{and} 30 \mu \mathrm{g} / \mathrm{ml}$ camphosulfonic acid as internal standards) and incubating at $-30^{\circ} \mathrm{C}$ for $1 \mathrm{~h}$. For each sample, $1.2 \mathrm{ml}$ of solvent containing extracted metabolites was then mixed with $600 \mu$ 1 ultrapure water, vortexed briefly, and then centrifuged at $9,390 \times g$ at $4{ }^{\circ} \mathrm{C}$ for 3 min to separate the aqueous and organic phases. The aqueous phase containing hydrophilic metabolites was filtered through syringe-mounted PTFE filter units (pore size $0.20 \mu \mathrm{m}$; Millipore, MA, USA), and then $350 \mu \mathrm{l}$ and $700 \mu \mathrm{l}$ were taken for GC-MS and LC-MS analysis, respectively. Residual organic solvent was removed from the samples by centrifugal concentration, and then the samples were freezedried overnight and stored at $-80^{\circ} \mathrm{C}$ until analysis.

For GC-MS analysis, extracted metabolites were first derivatized by oximation (addition of $100 \mu \mathrm{l} 20 \mathrm{mg} / \mathrm{ml}$ methoxyamine hydrochloride in pyridine, 1,200 r.p.m. for $90 \mathrm{~min}$ at $30^{\circ} \mathrm{C}$ ) and silylation (addition of $50 \mu \mathrm{l}, N$-methyl- $N$-(trimethylsilyl) trifluoroacetamide (MSTFA), 1,200 r.p.m. for $30 \mathrm{~min}$ reaction at $\left.37^{\circ} \mathrm{C}\right)$. The derivatized samples were analyzed on a GC-MS-QP2010 Ultra (Shimadzu, Kyoto, Japan) with InertCap 5MS/NP column ( $0.25 \mathrm{~mm}$ ID x $30 \mathrm{~m}, \mathrm{df}=0.25 \mu$ $\mathrm{m}$; GL Sciences, Tokyo, Japan). An alkane standard mix (C8 to C40) was injected before sample analysis for calculating retention indices. Peak detection, baseline correction and retention time alignment were performed using MetAlign followed by automated peak identification with AIoutput 2 ver.1.29. For each sample, peak intensities were normalized to the internal standard (ribitol).

For LC-MS analysis, extracted metabolites were resuspended in $35 \mu$ 1 ultrapure water and analyzed on a Shimadzu Nexera UHPLC system coupled to LC-MS 8030 Plus (Kyoto, Japan) using a Mastro C18 reversed phase HPLC column $(150 \mathrm{~mm} \times 2.1 \mathrm{~mm}$, particle size $3 \mu \mathrm{m}$; Shimadzu, Kyoto, Japan $)$ operated in multiple reaction monitoring (MRM) mode. The mobile phases were $10 \mathrm{mM}$ tributylamine and $15 \mathrm{mM}$ acetic acid in water (A) and methanol (B). Peak identification and quantitation of peak areas were done using the LabSolutions software (Shimadzu, Kyoto, Japan).

Statistical analysis. In all figures, error bars represent s.d. of the mean unless otherwise noted. Growth curves, metabolomic analysis and specific growth rates were measured from $n=3$ biologically independent samples. Hydrogen peroxide and ammonia formation assays were performed using purified SpeC G655S enzyme with $n=3$ independent repeats. Gene expression using RT-qPCR was measured on $n=3$ biologically independent samples. Calculations for RT-qPCR were calculated using $\Delta \Delta \mathrm{Cq}$ method by Bio-Rad CFX Manager 2.0. Enzyme kinetics of YdfG, SpeC, Upp and GabT were measured from purified protein and measurements were taken with $n=3$ independent repeats. To determine product formation from GabT and SpeC variants on different substrates using GC-MS, the product of three independent assays were measured to reveal similar mass spectra of the enzyme product.

\section{Data availability}

The data that support the findings of this study are available from the corresponding author upon reasonable request. All genomic sequences are available at NCBI under BioProject ID PRJNA485586.

\section{References}

23. Baba, T. et al. Construction of Escherichia coli K-12 in-frame, single-gene knockout mutants: the Keio collection. Mol. Syst. Biol. 2, 2006.0008 (2006).

24. Espah Borujeni, A., Channarasappa, A. S. \& Salis, H. M. Translation rate is controlled by coupled trade-offs between site accessibility, selective RNA unfolding and sliding at upstream standby sites. Nucleic Acids Res. 42, 2646-2659 (2014).

25. Salis, H. M., Mirsky, E. A. \& Voigt, C. A. Automated design of synthetic ribosome binding sites to control protein expression. Nat. Biotechnol. 27, 946-950 (2009).

26. Datsenko, K. A. \& Wanner, B. L. One-step inactivation of chromosomal genes in Escherichia coli K-12 using PCR products. Proc. Natl Acad. Sci. USA 97, 6640-6645 (2000).

27. Degnen, G. E. \& Cox, E. C. Conditional mutator gene in Escherichia coli: isolation, mapping, and effector studies. J. Bacteriol. 117, 477-487 (1974). 
28. Livingston, D. Deoxyribonucleaic acid polymerase III of Escherichia coli. J. Biol. Chem. 250, 489-497 (1975).

29. Jensen, K. F. \& Mygind, B. Different oligomeric states are involved in the allosteric behavior of uracil phosphoribosyltransferase from Escherichia coli. Eur. J. Biochem. 240, 637-645 (1996).

30. Wernick, D. G., Pontrelli, S. P., Pollock, A. W. \& Liao, J. C. Sustainable biorefining in wastewater by engineered extreme alkaliphile Bacillus marmarensis. Sci. Rep. 6, 20224 (2016).

31. Canellakis, E. S., Paterakis, A. A., Huang, S. C., Panagiotidis, C. A. \& Kyriakidis, D. A. Identification, cloning, and nucleotide sequencing of the ornithine decarboxylase antizyme gene of Escherichia coli. Proc. Natl Acad. Sci. USA 90, 7129-7133 (1993).

32. Choi, K. Y., Wernick, D. G., Tat, C. A. \& Liao, J. C. Consolidated conversion of protein waste into biofuels and ammonia using Bacillus subtilis. Metab. Eng. 23, 53-61 (2014).

33. Applebaum, D. M., Dunlap, J. C. \& Morris, D. R. Comparison of the biosynthetic and biodegradative ornithine decarboxylases of Escherichia coli. Biochemistry 16, 1580-1584 (1977).
34. Smart, K. F., Aggio, R. B. M., Van Houtte, J. R. \& Villas-Bôas, S. G. Analytical platform for metabolome analysis of microbial cells using methyl chloroformate derivatization followed by gas chromatography-mass spectrometry. Nat. Protoc. 5, 1709-1729 (2010).

35. Li, H. \& Durbin, R. Fast and accurate long-read alignment with BurrowsWheeler transform. Bioinformatics 26, 589-595 (2010).

36. McKenna, A. et al. The Genome Analysis Toolkit: a MapReduce framework for analyzing next-generation DNA sequencing data. Genome Res. 20, 1297-1303 (2010).

37. Cingolani, P. et al. A program for annotating and predicting the effects of single nucleotide polymorphisms, SnpEff: SNPs in the genome of Drosophila melanogaster strainw1118; iso-2; iso-3. Fly (Austin) 6 , 80-92 (2012).

38. Untergasser, A. et al. Primer3-new capabilities and interfaces. Nucleic Acids Res. 40, e115 (2012).

39. Liu, M. et al. Global transcriptional programs reveal a carbon source foraging strategy by Escherichia coli. J. Biol. Chem. 280, 15921-15927 (2005) 


\section{Reporting Summary}

Nature Research wishes to improve the reproducibility of the work that we publish. This form provides structure for consistency and transparency in reporting. For further information on Nature Research policies, see Authors \& Referees and the Editorial Policy Checklist.

\section{Statistical parameters}

When statistical analyses are reported, confirm that the following items are present in the relevant location (e.g. figure legend, table legend, main text, or Methods section).

n/a Confirmed

$\bigotimes$ The exact sample size $(n)$ for each experimental group/condition, given as a discrete number and unit of measurement

$\bigotimes$ An indication of whether measurements were taken from distinct samples or whether the same sample was measured repeatedly

The statistical test(s) used AND whether they are one- or two-sided

$\triangle$ Only common tests should be described solely by name; describe more complex techniques in the Methods section.

$\bigotimes \square$ A description of all covariates tested

Х $\square$ A description of any assumptions or corrections, such as tests of normality and adjustment for multiple comparisons

A full description of the statistics including central tendency (e.g. means) or other basic estimates (e.g. regression coefficient) AND variation (e.g. standard deviation) or associated estimates of uncertainty (e.g. confidence intervals)

$\otimes \square$ For null hypothesis testing, the test statistic (e.g. $F, t, r$ ) with confidence intervals, effect sizes, degrees of freedom and $P$ value noted Give $P$ values as exact values whenever suitable.

Х $\square$ For Bayesian analysis, information on the choice of priors and Markov chain Monte Carlo settings

Х $\square$ For hierarchical and complex designs, identification of the appropriate level for tests and full reporting of outcomes

Х $\square$ Estimates of effect sizes (e.g. Cohen's $d$, Pearson's $r$ ), indicating how they were calculated

$\varnothing$ Clearly defined error bars

$\triangle$ State explicitly what error bars represent (e.g. SD, SE, CI)

Our web collection on statistics for biologists may be useful.

\section{Software and code}

\section{Policy information about availability of computer code}

Data collection

RT-qPCR measurements were performed using Bio-Rad CFX Manager 2.0.

Data analysis

RT-qPCR analysis was performed using Bio-Rad CFX Manager 2.0. Gene expression and normalization was calculated using the ??Cq method.

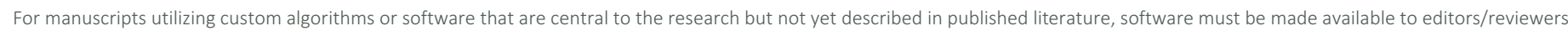

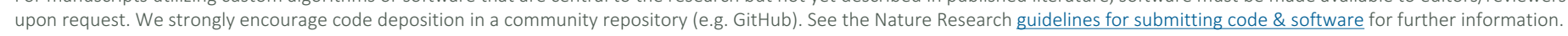

\section{Data}

Policy information about availability of data

All manuscripts must include a data availability statement. This statement should provide the following information, where applicable:

- Accession codes, unique identifiers, or web links for publicly available datasets

- A list of figures that have associated raw data

- A description of any restrictions on data availability

The data that support the findings of this study are available from the corresponding author upon reasonable request. 
Please select the best fit for your research. If you are not sure, read the appropriate sections before making your selection.

$\bigotimes$ Life sciences $\quad \square$ Behavioural \& social sciences $\quad \square$ Ecological, evolutionary \& environmental sciences

For a reference copy of the document with all sections, see nature.com/authors/policies/ReportingSummary-flat.pdf

\section{Life sciences study design}

All studies must disclose on these points even when the disclosure is negative.

Sample size No sample size calculations were performed. Sample sizes for all experiments were performed using triplicates. This was determined as appropriate based on numbers typically used in similar experiments reported in the literature.

Data exclusions No data was exluded

Replication All experiments were successfully repeated as noted in the statistical information section in the methods section.

Randomization Individual colonies for experimental analysis were chosen at random

Blinding Due to different nutritional and/or antibiotic requirements for different strains, no blinding was performed in this research.

\section{Reporting for specific materials, systems and methods}

Materials \& experimental systems

$\mathrm{n} / \mathrm{a}$ Involved in the study

\ $\square$ Unique biological materials

Х $\square$ Antibodies

Х $\square$ Eukaryotic cell lines

Х $\square$ Palaeontology

$\square$ Animals and other organisms

Х $\square$ Human research participants
Methods

$\mathrm{n} / \mathrm{a}$ Involved in the study

X $\square$ chip-seq

Х $\square$ Flow cytometry

Х $\square$ MRI-based neuroimaging 